\title{
Ras-Related Protein Ral-A
}

National Cancer Institute

\section{Source}

National Cancer Institute. Ras-Related Protein Ral-A. NCI Thesaurus. Code C18291.

Ras-related protein Ral-A (206 aa, 24 kDa) is encoded by the human RALA gene. This protein plays a role in GT Pase-mediated signaling. 